\title{
Article
}

\section{A Twin Study of Cigarette and Snus Initiation and Quantity of Use in Norwegian Adult Twins}

\author{
Kenneth S. Kendler ${ }^{1,2,3}$, Steven H. Aggen ${ }^{1,2}$, Nathan Gillespie ${ }^{1,2}$, Nikolai Czajkowski ${ }^{4,5}$, Eivind Ystrom ${ }^{4,5}$ and \\ Ted Reichborn-Kjennerud ${ }^{4,6}$ \\ ${ }^{1}$ Virginia Institute for Psychiatric and Behavioral Genetics, Richmond, VA, USA, ${ }^{2}$ Department of Psychiatry, Virginia Commonwealth University, Richmond, VA, \\ USA, ${ }^{3}$ Department of Human and Molecular Genetics, Virginia Commonwealth University, Richmond, VA, USA, ${ }^{4}$ Department of Mental Disorders, Norwegian \\ Institute of Public Health, Oslo, Norway, ${ }^{5}$ Department of Psychology, University of Oslo, Oslo, Norway and ${ }^{6}$ Institute of Clinical Medicine, University of Oslo, Oslo, \\ Norway
}

\begin{abstract}
While snus has been the focus of increasing public health interest, twin studies have examined neither sources of individual variation for its use nor the sources of resemblance between snus and cigarette use. Twins from the Norwegian Institute of Public Health Panel were assessed by self-report questionnaire for the initiation of regular use and maximal quantity used for snus and cigarettes. Twin modeling was performed using OpenMx on data from 2767 twins including 856 complete pairs. Fitting univariate twin models produced similar results for cigarette initiation and quantity with estimates of additive genetic, shared environmental and unique environmental effects of approximately $77 \%$, $0 \%$ and $23 \%$, respectively. Estimates of snus initiation and quantity were, respectively, approximately $53 \%, 26 \%$ and $21 \%$. Joint analyses suggested that the genetic, shared environmental and unique environmental correlations between cigarette and snus initiation and quantity were $+.82,0$ and +.42 , respectively. However, these results could not be statistically distinguished from a model which postulated that resemblance between cigarette initiation and quantity resulted from genetic and unique environmental correlations of +.47 and +.43 . Compared with cigarette initiation and quantity of use in Norwegian twins, the role of genes was less prominent and shared environment more prominent for initiation and quantity of use of snus. Joint analyses of both tobacco phenotypes suggested, but did not confirm definitively, that genetic risk factors for cigarette and snus use were similar but not identical, while shared environmental factors existed that were specific to snus use.
\end{abstract}

Keywords: Twins; tobacco; cigarettes; snus

(Received 6 November 2018; accepted 25 February 2019)

A substantial twin literature has examined the sources of individual differences in the initiation of cigarette use and the quantity of cigarettes consumed (Kendler et al., 2008; Li et al., 2003; Maes et al., 2017; Sartor et al., 2015; Sullivan \& Kendler, 1999; Vink et al., 2005). These studies provide strong support for an important role of genetic factors in twin resemblance, with shared environmental effects tending to be stronger for initiation than quantity consumed and stronger in younger versus older samples. By comparison, noncigarette forms of tobacco use have been subject to far less attention in the genetic epidemiological literature. To our knowledge, only one prior study has compared the role of genetic and environmental factors in individual differences for multiple different forms of administration of tobacco (Schmitt et al., 2005). This study examined regular use of cigarettes, cigars, pipes, dip (moist snuff), and chewing tobacco. The relative role of genetic and environmental factors differed substantially across forms of administration. Twin similarity for regular cigarette and dip use was

\footnotetext{
Author for correspondence: Kenneth S. Kendler, Email: kenneth.kendler@vcuhealth. org

Cite this article: Kendler KS, Aggen SH, Gillespie N, Czajkowski N, Ystrom E, and Reichborn-Kjennerud T. (2019) A Twin Study of Cigarette and Snus Initiation and Quantity of Use in Norwegian Adult Twins. Twin Research and Human Genetics 22: 108-113, https://doi.org/10.1017/thg.2019.9
}

largely genetic in origin, while twin resemblance for chewing tobacco resulted from the effects of both genes and shared environment, and for pipes and cigars was entirely the result of the shared environment. However, these analyses did not examine what might be an even more interesting question: the level of similarity in the genetic and environmental risk factors for different forms of administration of tobacco?

We are aware of one prior twin study that has addressed this question for cannabis and cocaine (Agrawal et al., 2004) by fitting bivariate twin models to data on the use of two forms of cannabis (leaf marijuana and hashish) and two forms of cocaine (intranasal powder and inhaled crack). For both drugs, the analyses demonstrated a complete overlap of genetic factors and a very high correlation of shared environmental influences between the two forms of administration.

In this study, we examine the initiation of and quantity of use of cigarettes and snus (a type of moist powdered tobacco, typically held in the mouth between the lips and gums) in a populationbased Norwegian sample of adult twins. Snus has been a subject of increasing interest because of evidence that it may have less adverse health effects than cigarettes (Lee, 2013) and may be an effective substitute for cigarettes comparable in efficacy to nicotine patches (Hatsukami et al., 2016), and it may be more strongly 
associated with smoking cessation than smoking initiation (Furberg et al., 2005). Furthermore, despite declines in cigarette use, snus use is increasingly popular in Norway (Lund et al., 2017).

We first report analyses of the role of genetic and environmental factors in twin resemblance for initiation and frequency of use for these two forms of tobacco. Then, we report a bivariate analysis of cigarette and snus use to examine the degree to which similar genetic and environmental factors influence these two modes of administration of tobacco consumption. Consistent with the one prior twin study of forms of tobacco use (Schmitt et al., 2005), we predict differences in the sources of variance in liability to the use of cigarettes and snus.

\section{Methods}

\section{Participants}

Data for the twin analyses of lifetime regular cigarette and snus (a form of smokeless tobacco) use reported in this study came from the most recently completed data collection project conducted by the Norwegian Institute of Public Health (NIPH) Twin Panel (Nilsen et al., 2012). An internally developed self-report questionnaire was distributed via surface mail beginning in November 2015 to $N=6308$ invited/eligible twins with a last distribution occurring in June 2017. As of October 2017, $N=3862$ (61\%) twins had returned a questionnaire for processing. The total sample consisted of two different groups of twins. One was made up of twins who had been previously contacted and participated $(N=1916)$ in at least one of the previously conducted interviews (mean; $S D$ ) age $=43.1$ and 3.8 , range $=36-50$ ). These twins were originally identified through birth records (1967-1979) from the Norwegian National Medical Birth Registry $(N=15,370)$ and had not previously been asked about their cigarette and snus use. A second group $(N=1946)$ was made up of twins who agreed to be registered in the official Norway twin registry and were participating for the first time (age $=42.9$ [3.7], range $=36-49$ ). The breakdown by zygosity classifications in the full questionnaire sample is as follows: $1103 \mathrm{com}$ plete pairs: 202 monozygotic (MZ) males, 101 dizygotic (DZ) males, $344 \mathrm{MZ}$ females, $206 \mathrm{DZ}$ females, and 250 opposite sex pairs. For the tobacco analyses performed in this study, only same-sex MZ and DZ pairs were included, given the low power of qualitative sex-effect analyses with the limited number of available opposite-sex pairs. This translates into $N=1037$ (549 complete and 491 singleton) same sex MZ pairs and $N=871$ (307 complete and 564 singleton) same-sex DZ pairs.

\section{Questionnaire Data}

The Norwegian Institute of Public Health self-report questionnaire (NIPH-SRQ) included two separate sections asking about lifetime regular tobacco use and information about tobacco use-related behaviors. One section enquired about lifetime regular cigarette use and quantity consumed. Responses to the item "Have you ever smoked cigarettes regularly for at least one month?" (yes/no coded as 1-0) served as data for the tobacco phenotype of regular cigarette smoking that we define here as initiation. A second section enquired about the use of snus, "Have you ever used snus regularly for at least a month?" (yes/no coded as 1-0), providing data for the second tobacco phenotype of ever regular use of snus, which we termed here as snus initiation.

For both types of tobacco, twins were also asked about the amount of tobacco they used when their usage was the heaviest. For cigarettes, responses to the item "When you smoked the most,

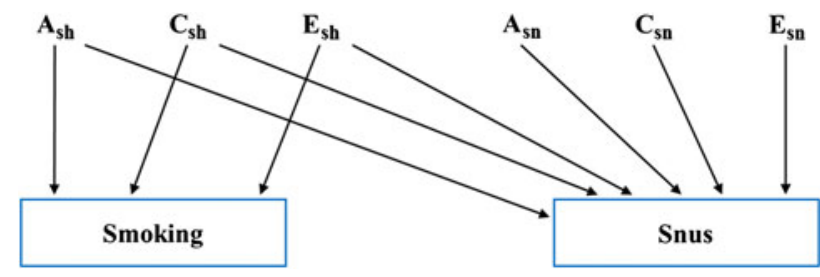

Fig. 1. A bivariate Cholesky decomposition for smoking and snus-related phenotypes. The model contains "upstream" variables reflecting shared additive genetic $\left(A^{2}{ }_{s h}\right)$, shared environmental $\left(\mathrm{C}^{2}{ }_{\text {sh }}\right)$ and individual-specific environmental effects $\left(\mathrm{E}^{2}{ }_{\mathrm{sh}}\right)$, where the subscript sh stands for shared, and downstream variables that are specific to snus: $A^{2}{ }_{s n}, C^{2}$ sn and $E_{s n}^{2}$, where the subscript sn stands for specific to snus.

on average, how many cigarettes did you smoke per day?" ranged from 0 to 80 cigarettes per day. Because the frequency distribution of the reported cigarettes per day variable was expectedly uneven, we constructed a three-category ordinal variable for data analysis: $1=0-10,2=11-20$, and $3=20$ or more. The quantity used variable for snus, "When you used snus the most, how many times per day did you use it?", had three response options: 1-7 times, 8-12 times, and 13 or more. If either of the cigarette or snus quantity used variables were missing but their corresponding ever used regularly variables were checked as "no" (coded zero), the respective quantity used variables were also set to zero, forming an "abstainer" category.

\section{Statistical Analyses}

We utilized standard twin modeling techniques (Neale \& Cardon, 1992) in which the latent liability to cigarette and snus initiation and quantity of use was decomposed into three components: $\mathrm{a}^{2}$ or additive genetic effects, $c^{2}$ or shared environmental effects, and $\mathrm{e}^{2}$ or individual specific effects. In these designs, the latter are confounded with measurement error. We began with univariate analyses of our four phenotypes.

We then utilized bivariate Cholesky decompositions to estimate genetic and environmental components common to both cigarette and snus, and components specific to snus. As seen in Figure 1, a Cholesky decomposition contains both $\mathrm{a}^{2}{ }_{\mathrm{sh}}, \mathrm{c}^{2}{ }_{\text {sh }}$ and $\mathrm{e}^{2}$ sh, where the subscript sh stands for shared between cigarettes and snus, and $\mathrm{a}^{2}{ }_{\text {sn }}, \mathrm{c}^{2}$ sn and $\mathrm{e}^{2}$ sn where the subscript sn stands for specific to snus. Cholesky decompositions were carried out separately for (1) binary cigarette and snus initiation and (2) ordinal scaled maximal lifetime daily cigarette and snus use. Each decomposition was fitted to a two-group MZ/DZ twin data structure. Empirical optimization was carried out using a full information maximum likelihood (FIML) raw data method treating the observed tobacco variables as categorical as implemented in the version 2.7.16 free OpenMx package (Neale et al., 2016) and run in the R 3.4.1 (R Development Core Team, 2017) computing environment. Liability threshold models (Falconer, 1965) were fit to the binary and ordinal phenotype variables. The observed frequencies of the outcome variable categories are used to obtain estimates of the between category thresholds on an unobserved continuous liability response variable using a normal Gaussian Probit model. For example, for the cigarette initiation 0-1 binary variable, a single threshold is estimated on a hypothesized unobserved liability variable on which the twin1-twin2 MZ and DZ phenotypic correlations are estimated. To provide stability to the estimation of certain C path coefficients, lower bounds of .001 were included for the Cholesky path point estimates. The $-2 \log$ likelihood misfits for models with and without the lower boundary constraints were 
Table 1. Frequency and quantity of cigarette and snus use in our twin sample

\begin{tabular}{lccccc}
\hline Sex & $N$ & Cigarettes & Snus \\
\hline Initiation \pm SEM & $\begin{array}{c}\text { Cigarettes per day at } \\
\text { heaviest use (SD) }\end{array}$ & $\begin{array}{c}\text { Initiation } \pm \text { SEM } \\
\text { Number of uses per day } \\
\text { at heaviest use (SD) }\end{array}$ & $\begin{array}{c}1.92 \pm 0.01 \\
(0.78)\end{array}$ \\
\hline Males & 1538 & $0.39 \pm 0.01$ & $17.1(8.5)$ & $0.06 \pm 0.01$ & $1.75(0.69)$ \\
\hline
\end{tabular}

Table 2. Twin correlations, univariate model fits and parameter estimates and $95 \% \mathrm{Cls}$ for full models for cigarettes and snus initiation and quantity used

\begin{tabular}{|c|c|c|c|c|c|c|c|c|c|}
\hline \multirow[b]{2}{*}{ Phenotype } & \multirow[b]{2}{*}{ Form } & \multirow{2}{*}{$\begin{array}{c}\text { Correlation } \\
\text { MZ pairs (SE) }\end{array}$} & \multirow{2}{*}{$\begin{array}{c}\text { Correlation } \\
\text { DZ pairs (SE) }\end{array}$} & \multicolumn{3}{|c|}{ Model fit AIC } & \multirow[b]{2}{*}{ a } & \multirow[b]{2}{*}{ c } & \multirow[b]{2}{*}{ e } \\
\hline & & & & ACE & $\mathrm{AE}$ & CE & & & \\
\hline Cigarettes & Quantity used & $.74 \pm .03$ & $.32 \pm .08$ & -94 & -96 & -63 & $0.86[0.70,0.89]$ & $0.00[0.00,0.47]$ & $0.51[0.46-0.57]$ \\
\hline Snus & Initiation & $.81 \pm .04$ & $.54 \pm .11$ & -3233 & -3233 & -3228 & $0.72[0.32,0.93]$ & $0.54[0.00,0.83]$ & $0.44[0.34,0.53]$ \\
\hline
\end{tabular}

identical. We evaluated the results of our model fitting using Akaike's Information Criterion (Akaike, 1987) where we picked the model with the lowest value, as it represents the best balance between explanatory power and parsimony. We report here genetic and environmental correlations between snus and cigarette initiation and frequency of use.

\section{Results}

\section{Descriptive Results}

As seen in Table 1, females initiated regular smoking slightly more frequently than males ( 43 to $39 \%$; $p=.51$ ), but as smokers, men consumed a significantly higher number of cigarettes per day at their time of heaviest use (17.1 vs. $14.1 ; p<.001$; Figure 2$)$. Sex differences were more marked for initiation of use of snus which was seen in $32 \%$ of men and $6 \%$ of women $(p<.0001)$. Among the snus users, consumption was somewhat greater in males than females $(p=.08)$. We had in our sample 307 men and 121 women who report using both cigarettes and snus.

\section{Univariate Modeling}

Table 2 presents the tetrachoric correlations in $\mathrm{MZ}$ and $\mathrm{DZ}$ twin pairs for initiation of regular cigarette and snus use and the polychoric correlations for the quantity of cigarettes and snus consumed at time of heaviest use. Correlations for all four of these traits were quite high in $\mathrm{MZ}$ twins, ranging from +.74 to +.82 . In $\mathrm{DZ}$ pairs, correlations for both initiation and quantity of cigarette use were around +.35 , very close to one-half the correlation observed in MZ pairs. This is the pattern expected if twin resemblance arises solely from genetic factors. By contrast, correlations for both initiation and quantity of snus use in DZ twins were substantially more than half that observed in MZ twins suggesting the importance of shared family environment.

Table 2 presents AIC values for the three standard twin models: the full or ACE model and two restricted models which assume that all twin resemblance results from genetic effects (the $\mathrm{AE}$ model) or from shared environmental effects (CE model). As expected from the pattern of twin correlations, for both initiation and quantity of cigarettes, the AE model provided the best fit by AIC. By contrast, for snus initiation and quantity, the fit was the same for the ACE and AE models.

Table 2 also provides parameter estimates (and 95\% CIs) for A, $\mathrm{C}$ and $\mathrm{E}$ from the full or ACE model. As expected, the values of $\mathrm{c}^{2}$ for cigarette initiation and quantity were both estimated at zero. Estimates of $\mathrm{a}^{2}$ and $\mathrm{e}^{2}$ for these two phenotypes were, respectively, 0.79 and 0.21 , and 0.74 and 0.26 .

By contrast, estimates of $\mathrm{c}^{2}$ for snus initiation and quantity were appreciable and, although known imprecisely, were significantly greater than zero. For snus initiation, estimates of $\mathrm{a}^{2}, \mathrm{c}^{2}$ and $\mathrm{e}^{2}$ were, respectively, $0.51,0.30$ and 0.19 . Parallel values for quantity of snus used were $0.54,0.23$ and 0.23 .

\section{Bivariate Modeling}

We report the results of a Cholesky decomposition analysis in Table 3, where we defined cigarette initiation or frequency of use as the upstream or independent variable and snus initiation or frequency as the downstream or dependent variable. We also present genetic and environmental correlations estimated from these results. We first examine cigarette and snus initiation. Our focus in the interpretation of these models is on the sources of genetic and environmental effects that are predicted to be specific to snus. The ACE and $\mathrm{AE}$ model produced similar fits by AIC (slightly better for the $\mathrm{AE}$ models), and so, we present results from both models. The ACE model shows a substantial genetic cross-path from cigarette initiation to snus initiation but no shared environmental effects common to the two forms of tobacco use. In this model, familial factors specific to snus initiation use were largely shared environmental origin with only moderate contribution from genes. Unique environmental factors also contributed moderately to snus-specific effects. The ACE model predicted a substantial genetic correlation between cigarette and snus initiation of +.82 but no shared environmental correlation and a modest unique environmental correlation $(+.42)$. The AE model produced quite different results. Here, familial effects that impacted only on snus initiation were entirely genetic. In this model, the genetic correlation between cigarette and snus initiation was modest and estimated at +.47 . 


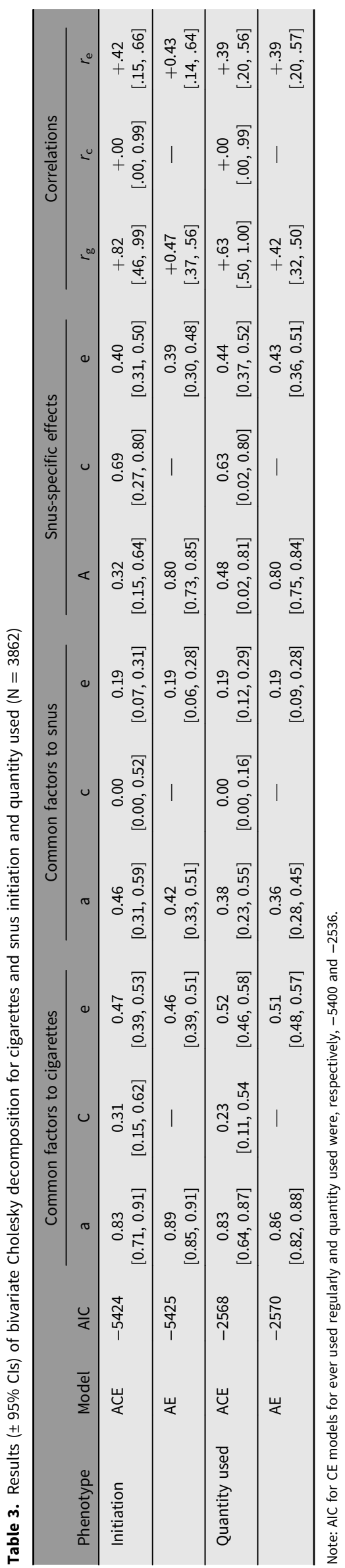

The pattern of results was similar for quantity of use of cigarettes and snus. The AE model fit slightly better than the ACE model. As with initiation, the ACE model predicted that a preponderance of the familial effects specific to the quantity of snus consumed were largely shared environmental with a moderate contribution of snus-specific genetic effects. The genetic correlation was estimated to be relatively high: +.63 . The AE model presents a quite different picture where only genes were responsible for the familial effects specific to snus use. This model estimated the genetic correlation between the quantity of use of cigarettes and snus was relatively modest and estimated at +.42 .

\section{Discussion}

This paper had two interrelated goals. We first examined in the same population-based Norwegian twin sample the role of genetic and environmental factors in twin resemblance for initiation and frequency of use for cigarettes and snus. Then, we examined, for the first time to our knowledge, a bivariate analysis of cigarette and snus initiation and frequency of use to examine the degree to which similar or different genetic and environmental factors influence these two modes of tobacco self-administration. We review these results in turn.

First, we found that in adulthood, initiation of regular cigarette use and quantity of use were both highly heritable traits with no evidence of shared environmental effects. Our estimates of heritability $(79 \%$ and $74 \%)$ are toward the upper range of those previously reported (Li et al., 2003; Sullivan \& Kendler, 1999) and our lack of evidence for shared environmental effects is somewhat unusual. However, in the 17 twin studies of smoking initiation in adults reviewed by Sullivan and Kendler (1999), 4 studies found estimates of heritabilities of $>70 \%$ and 3 reported estimates of $c^{2}<$ $10 \%$. In the presence of substantial genetic effects, our sample is not well-powered to detect small shared environmental influences (Neale et al., 1994).

Second, the pattern of results for snus initiation and frequency of use differed meaningfully from that found for cigarettes in the Norwegian population. Heritability estimates for snus were lower, and unlike for cigarettes, there was clear evidence for shared environmental effects. These findings are broadly replicated by the one previous twin study of multiple forms of tobacco use performed in Virginia where estimates of shared environmental effects were lower for cigarettes than those found for all the other forms of tobacco, including cigars, pipes, dips and chewing tobacco (Schmitt et al., 2005). One plausible explanation for these findings is that the universal availability and widespread use of cigarettes reduces familial-environmental influences on its use, while for the rarer forms of tobacco, within-family social learning effects (Bandura, 1986) play a stronger role in decisions to initiate use.

Third, we examined, using bivariate Cholesky decomposition, the nature of the sharing of genetic and familial-environmental risk factors for cigarette and snus initiation and quantity of use. The interpretation of these results is somewhat more problematic than those for the univariate analyses. We present results both from the ACE and AE models that tell rather different stories about the causes of resemblance for cigarette and snus use. The slightly better-fitting AE models suggest that genetic effects were the sole source of familial effects specific to snus initiation and quantity of use. By contrast, the ACE models suggest that the bulk of familial effects specific to snus was environmental in origin, with a modest contribution of genetic effects. 


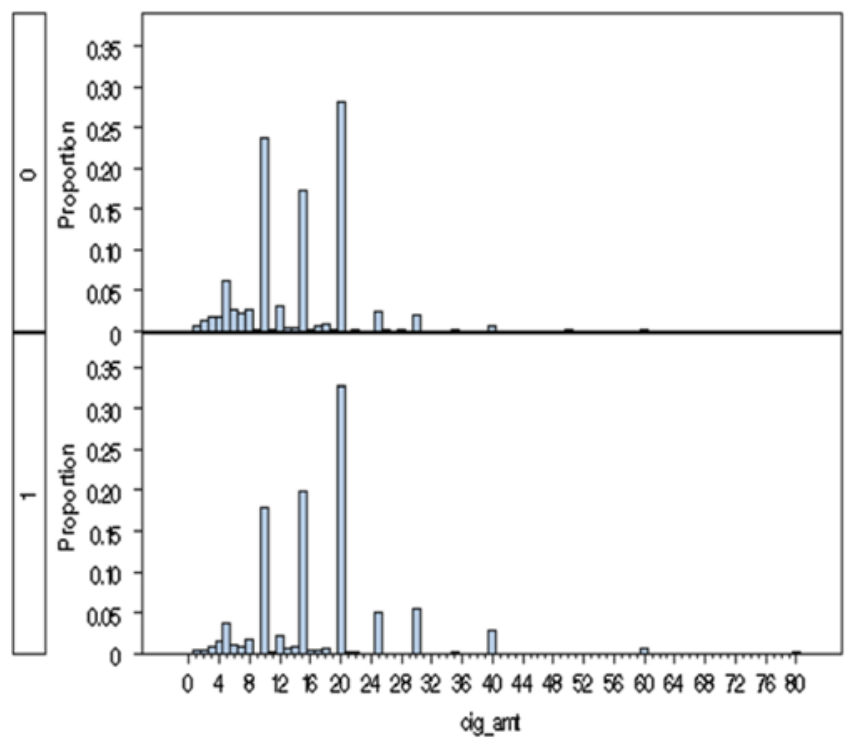

Fig. 2. A histogram of the frequency of reported cigarettes per day at time of heaviest use in females (top half) and males (bottom half). Note: $1=$ males, $0=$ females.

Although not fitting quite as well, we favor results from the ACE model for three reasons. First, when power in twin analyses is low, as it is in this case with our modest sample size, Sullivan and Eaves (2002) have convincingly argued that results from the full model are likely to be more accurate than those for the best-fit model. Second, this is particularly true when trying to estimate accurately the shared environmental contribution in the presence of robust genetic effects, which can be done with high confidence only in quite large twin samples (Neale et al., 1994). Third, both an examination of the twin correlations and the univariate analyses provide rather convincing evidence for shared environmental effects for snus initiation and quantity of use. This makes suspect a bivariate model which, despite its good statistical fit, sets these effects to zero.

It is interesting to compare our findings with prior twin analyses of the use of different forms of cannabis (leaf marijuana and hashish) and cocaine (intranasal powder and inhaled crack; Agrawal et al., 2004). For both drugs, the analyses showed a complete overlap of genetic factors and a nearly complete overlap of shared environmental influences on the two forms of administration. This report and the one previous study of this question (Schmitt et al., 2005) suggest that the factors governing use of various forms of tobacco differ in ways that are not seen for the use of the various forms of cannabis and cocaine. Perhaps the much longer history of tobacco use and the associated strength of cultural factors impacting on its consumption (Courtwright, 2001) are responsible for these observed differences

\section{Limitations}

These results should be interpreted in the context of several potentially important methodological limitations. First, our results are only relevant for the Norwegian population. Whether they will extrapolate to other populations is an empirical question. Second, despite a reasonable sample size of twins, our power was limited, especially in distinguishing the ACE and AE models. Simulations presented in Neale et al. (1994) demonstrate that with the sample sizes and trait frequencies available to us, we would be well powered only to detect $\mathrm{c}^{2}$ components responsible for more than $35 \%$ of phenotypic variance. For this paper, we performed simulations addressing our ability to detect subtler levels of $\mathrm{c}^{2}$ of $5 \%$ of total variance (Verhulst, 2017). At appropriate levels of prevalence and heritability, over 10,000 twin pairs are required to detect $c^{2}$ with $80 \%$ power.

Third, given our limited ability to distinguish key hypotheses in our entire sample, we were also insufficiently powered to be able to evaluate and test for qualitative sex effects. Substantial efforts were taken, and we were unable to obtain stable results. We tested our power formally (Verhulst, 2017). With our sample sizes, we could expect to reject a model assuming a genetic correlation of unity between the sexes with $80 \%$ power only with unrealistically small true genetic correlations of $\leq .20$. Fourth, we did not include age moderation in our analyses. However, across our relatively narrow age range (ages 36-50), prevalence of cigarette and snus use was relatively stable.

Financial support. This project was supported by Research Council of Norway grants 196148 and 226985, and NIH grant RO1DA037558. Previous analysis of twin data from this project was in part supported by grant MH- 068643 from the National Institutes of Health and previous data collection by grants from the Norwegian Research Council, the Norwegian Foundation for Health and Rehabilitation, the Norwegian Council for Mental Health, the Borderline Foundation and the European Commission.

Conflict of interest. None of the authors have any conflicts of interest to declare.

Ethical standards. The authors assert that all procedures contributing to this work comply with the ethical standards of the relevant national and institutional committees on human experimentation and with the Helsinki Declaration of 1975 , as revised in 2008 .

\section{References}

Agrawal, A., Prescott, C. A., \& Kendler, K. S. (2004). Forms of cannabis and cocaine: A twin study. American Journal of Medical Genetics Part B Neuropsychiatric Genetics, 129B, 125-128.

Akaike, H. (1987). Factor analysis and AIC. Psychometrika, 52, 317-332.

Bandura, A. (1986). Social foundations of thought and action: A social cognitive theory. Englewood Cliffs, NJ: Prentice-Hall.

Courtwright, D. T. (2001). Forces of habit: Drugs and the making of the modern world. Cambridge, MA: Harvard University Press.

Falconer, D. S. (1965). The inheritance of liability to certain diseases, estimated from the incidence among relatives. Annals of Human Genetics, 29, 51-76.

Furberg, H., Bulik, C. M., Lerman, C., Lichtenstein, P., Pedersen, N. L., \& Sullivan, P. F. (2005). Is Swedish snus associated with smoking initiation or smoking cessation? Tobacco Control, 14, 422-424.

Hatsukami, D. K., Severson, H., Anderson, A., Vogel, R. I., Jensen, J., Broadbent, B., ... Hecht, S. S. (2016). Randomised clinical trial of snus versus medicinal nicotine among smokers interested in product switching. Tobacco Control, 25, 267-274.

Kendler, K. S., Schmitt, E., Aggen, S. H., \& Prescott, C. A. (2008). Genetic and environmental influences on alcohol, caffeine, cannabis, and nicotine use from early adolescence to middle adulthood. Archives of General Psychiatry, 65, 674-682.

Lee, P.N. (2013). Epidemiological evidence relating snus to health - An updated review based on recent publications. Harm Reduction Journal, 10, 36.

Li, M. D., Cheng, R., Ma, J. Z., \& Swan, G. E. (2003). A meta-analysis of estimated genetic and environmental effects on smoking behavior in male and female adult twins. Addiction, 98, 23-31.

Lund, K. E., Vedoy, T. F., \& Bauld, L. (2017). Do never smokers make up an increasing share of snus users as cigarette smoking declines? Changes in smoking status among male snus users in Norway 2003-15. Addiction, $112,340-348$.

Maes, H. H., Prom-Wormley, E., Eaves, L. J., Rhee, S. H., Hewitt, J. K., Young, S., ... Neale, M. C. (2017). A genetic epidemiological mega analysis of smoking initiation in adolescents. Nicotine and Tobacco Research, 19, 401-409. 
Neale, M. C., \& Cardon, L. R. (1992). Methodology for genetic studies of twins and families. Dordrecht, Netherlands: Kluwer Academic Publishers B.V.

Neale, M. C., Eaves, L. J., \& Kendler, K. S. (1994). The power of the classical twin study to resolve variation in threshold traits. Behavior Genetics, 24, 239-258.

Neale, M. C., Hunter, M. D., Pritikin, J. N., Zahery, M., Brick, T. R., Kirkpatrick, R. M., ... Boker, S. M. (2016). OpenMx 2.0: Extended structural equation and statistical modeling. Psychometrika, 81, 535-549.

Nilsen, T. S., Brandt, I., Magnus, P., \& Harris, J. R. (2012). The Norwegian twin registry. Twin Research and Human Genetics, 15, 775-780.

R Development Core Team. (2017). R: A language and environment for statistical computing. Vienna, Austria: R Foundation for Statistical Computing.

Sartor, C. E., Grant, J. D., Agrawal, A., Sadler, B., Madden, P. A., Heath, A. C., \& Bucholz, K. K. (2015). Genetic and environmental contributions to initiation of cigarette smoking in young African-American and EuropeanAmerican women. Drug and Alcohol Dependence, 157, 54-59.

Schmitt, J. E., Prescott, C. A., Gardner, C. O., Neale, M. C., \& Kendler, K. S. (2005). The differential heritability of tobacco use based on method of administration: A twin study. Twin Research and Human Genetics, 8, 60-62.

Sullivan, P. F., \& Eaves, L. J. (2002). Evaluation of analyses of univariate discrete twin data. Behavior Genetics, 32, 221-227.

Sullivan, P. F., \& Kendler, K. S. (1999). The genetic epidemiology of smoking. Nicotine and Tobacco Research, 1, S51-S57.

Verhulst, B. (2017). A power calculator for the classical twin design. Behavior Genetics, 47, 255-261.

Vink, J. M., Willemsen, G., \& Boomsma, D. I. (2005). Heritability of smoking initiation and nicotine dependence. Behavior Genetics, 35, 397-406. 